\title{
On singular varieties having an extremal secant line
}

\author{
Marie-Amélie Bertin \\ 12 rue des Bretons, \\ 94700 Maisons-Alfort \\ FRANCE \\ marie.bertin@parisfree.com \\ mabertin@math.jussieu.fr
}

August 27, 2018

\section{Introduction}

Let $X$ be an irreducible non degenerate variety of degree $d$ and codimension $e$ in $\mathbb{P}^{r}$. Let us recall that, if $X$ is smooth, the regularity conjecture foresees that the Castelnuovo-Mumford regularity of $X$ is less or equal to $d-e+1$. This conjecture has been proved to hold for curves without the smoothness assumption by L.Gruson, R.Lazarsfeld and C.Peskine [6] and for smooth surfaces by H.Pinkham [10] (for the normality part of the conjecture) and R.Lazarsfeld [8]. In a previous article [1, we've extended Gruson, Lazarsfeld and Peskine's result to smooth scrolls over curves. In the curve case, Gruson, Lazarsfeld and Peskine give moreover a complete classification of curves of extremal regularity; they all posses an extremal secant line but the elliptic normal curves and the singular rational curves of degree $d$ in $\mathbb{P}^{d-1}$. In case the regularity conjecture holds, this leads us to believe that smooth varieties of extremal regularity of fixed dimension have an extremal secant line but a finite list of varieties. Varieties with an extremal secant line are moreover $(d-e)$-irregular, so they form a good test of the conjecture. This has lead us to classify the smooth ones and compute their regularity in a previous article [1. We gave a complete classification of the smooth varieties with an extremal 
secant line if $e \geq 2$; they are rational scrolls with an extremal secant line, the Veronese surface in $\mathbb{P}^{5}$ or its smooth projection to $\mathbb{P}^{4}$. This classification can be seen as a generalization of del Pezzo and Bertini 's classification of varieties of minimal degree, since such varieties correspond to varieties for which an extremal secant line is a bisecant line. Our classification results have been extended to a classification of smooth varieties having an extremal or next to extremal secant linear space $L$ by S.Kwak [7], under the extra assumption the extremal secant linear space $L$ cuts $X$ along a curvilinear 0-dimensional scheme, i.e. $\operatorname{dim}\left(L \cap \mathbb{T}_{p}(X)\right) \leq 1$ for all points $p \in L \cap X$. Independently, A. Noma [9] has recently found a generalization of Kwak's result:

Theorem 1.1 (B. $(x=0, k=1), \operatorname{Kwak}(x \leq 1)$, Noma). Let $X$ be a smooth, complex, irreducible, non degenerate variety of degree $d$ and codimension e of $\mathbb{P}^{r}$. Let $L$ be a $k$-dimensional linear space intersecting $X$ in a 0 -dimensional scheme of length $m$, such that $L \cap X$ is curvilinear. Assume that both $m \leq d-e+k-x$ and $e-k \geq x+1$. Then the sectional genus $\pi(X)$ satisfies $\pi(X) \leq x$.

Let us point out that Kwak's result (and ours) is more precise for it gives a classification result. In this note we'll see that this bound still holds for $x=0$ and $k=1$ without the smoothness assumption. In view of a classification theorem of varieties having an extremal secant line in the singular case, our previous paper [1] also stated a key result, that is unfortunately false in the singular case. In this note we correct it and deduce a classification of singular varieties of codimension $e \geq 2$ having an extremal secant line.

The first of these two key results in the singular case is: an extremal secant line to $X$ meets $X$ along smooth points. This is wrong; A. Noma kindly pointed out to us a very simple counterexample.

Example (Noma's counterexample). Let $C$ be a rational normal cubic curve and $X:=<q, C>$ a cone over $C$ in $\mathbb{P}^{4}=<q,<C>>$. A general line $l$ through $q$ meets $X$ with multiplicity 2 at $q$ and is an extremal secant line to $X$.

In this example one can find nonetheless an extremal secant line meeting $X$ along smooth points of $X$, namely, any bisecant line to $C$. This is a general phenomenon and we show in this note that we can correct our key lemma as follows: 
Theorem 1.2. Let $X$ be an irreducible, non degenerate variety of degree $d$ dimension $n \geq 2$ and codimension $e \geq 2$ in $\mathbb{P}^{r}$. Suppose that $X$ admits an extremal secant line $l$. Then, there exists an extremal secant line $l^{\prime}$ meeting $X$ along smooth points of $X$.

We deduce from this result the following classification theorem.

Theorem 1.3. Let $X$ be as in the previous theorem. Suppose that $e \geq 2$. Then the variety $X$ is either

1. a cone $\left\langle L, V>\right.$ over the Veronese surface $V$ in $\mathbb{P}^{5}$, where $L$ is a linear space of dimension $k \geq-1$

2. a cone $\left\langle L, V^{\prime}>\right.$ over $V^{\prime}$, the isomorphic projection of $V$ to $\mathbb{P}^{4}$, where $L$ is a linear space of dimension $k \geq-1$,

3. a cone $<L, X_{0}>$, where $X_{0}$ is a smooth rational scroll with an extremal secant line or a smooth rational curve with an extremal secant line, and $L$ a linear space of dimension $k \geq-1$.

\section{Basic facts and notations}

In this section we set up notations and recall the main results we use in the sequel. Let $X$ be a $n$-dimensional complex projective non-degenerate irreducible variety of degree $d$ in $\mathbb{P}^{r}$. We set $e:=r-n$, the codimension of $X$. In the rest of this article, the bracket $\langle\cdot\rangle$ denotes the linear span of the subvarieties of $\mathbb{P}^{r}$ listed in it.

A $k$-secant $m$-secant plane to $X$ is a $m$-plane $L$ such that $L \cap X$ is a 0 -dimensional scheme of degree at least $k$. We have the following classical result bounding $k$ :

Theorem 2.1 (linear section theorem). Let $X$ be as above. Let $\Lambda$ be a linear subspace of $\mathbb{P}^{r}$ of dimension $s \leq e$. Suppose that $\Lambda$ is not contained in $X$, then it cuts $X$ along a zero dimensional scheme of degree at most $d-e+s$.

A proof can be found for instance in [7].

In particular, a $k$-secant line $l$ to $X$ satisfies $k \leq d-e+1=: \delta$, so that we say that $l$ is an extremal secant line to $X$ if $k=d-e+1$. For example, varieties of minimal degree $Z$, i.e. such that $d=e+1$, of degree $d \geq 2$ have 
an extremal secant line; indeed, any bisecant line to $Z$ is an extremal secant line to $Z$.

Recall that a rational normal scroll of dimension $n \geq 2$ is the tautological embedding of some rank $n$ projective bundle over $\mathbb{P}^{1}$, say $\mathbb{P}\left(\mathcal{O}_{\mathbb{P}^{1}}\left(a_{1}\right) \oplus \cdots \oplus\right.$ $\left.\mathcal{O}_{\mathbb{P}^{1}}\left(a_{n}\right)\right)$ for some integers $0 \leq a_{1} \leq \cdots \leq a_{n}$. We denote such a scroll by $S\left(a_{1}, \cdots, a_{n}\right)$; it is a variety of degree $d=a_{1}+\cdots+a_{n}$, whose linear span is $\mathbb{P}^{d+n-1}$. It is therefore a variety of minimal degree. If $d>1$, the variety $S\left(a_{1}, \cdots, a_{n}\right)$ is smooth provided that $a_{1}>0$.

In case $n=2$, for all integer $k \geq 0$, we denote by $\mathbb{F}_{k}$ the projective $\mathbb{P}^{1}$-bundle $\mathbb{P}\left(\mathcal{O}_{\mathbb{P}^{1}} \oplus \mathcal{O}_{\mathbb{P}^{1}}(k)\right)$.

We have the following classification of varieties of minimal degree due to del Pezzo and Bertini.

Theorem 2.2 (del Pezzo, Bertini). Let $X$ be an irreducible, non-degenerate variety of codimension $e$ and degree $e+1$ in $\mathbb{P}^{r}$, i.e. bisecant lines are extremal secant lines to $X$. Then $X$ is a cone $<L, X_{0}>$, where $L$ is linear space of dimension $k \geq-1$ and $X_{0}$ is either

1. $(e=1)$ a linear space,

2. or $(e=2)$ a smooth quadric hypersurface,

3. or a smooth rational normal scroll,

4. or or the Veronese surface in $\mathbb{P}^{5}$.

A modern proof of this can be found in Eisenbud and Harris survey [5].

Let $\mathcal{I}_{X \mid \mathbb{P} r}$ be the ideal sheaf of $X$ in $\mathbb{P}^{r}$. The variety $X$ is said to be $k$-regular, for $k \in \mathbb{Z}$, if the following vanishing occur

$$
H^{i}\left(\mathcal{I}_{X \mid \mathbb{P}^{r}}(k-i)\right)=0, \quad \forall i \geq 1
$$

The $k$-regularity property of $X$ implies the $(k+1)$-regularity of $X$, so that one can define the Castelnuovo-Mumford regularity of $X$ as

$$
\operatorname{reg}(X):=\min \{k \in \mathbb{Z} \mid X \text { is } k-\text { regular }\}
$$

If $X$ is $k$-regular, the saturated ideal, $I_{X \mid \mathbb{P}^{r}}$, defining $X$ in $\mathbb{P}^{r}$ is generated by polynomials of degree $\leq k$; thus, if $X$ has a $k$-secant line, $\operatorname{reg}(X) \geq k$.

The regularity conjecture $([2,, 4])$ foresees that: $\operatorname{reg}(X) \leq d-e+1$ (Castelnuovo's bound). 
This conjecture has been proved to hold for curves by Gruson, Lazarsfeld and Peskine [6]. In this beautiful paper, they also completely classify $(d-e)$ irregular curves. This is a key result for the classification of varieties with an extremal secant line.

Theorem 2.3 (Gruson-Lazarsfeld-Peskine, 1983). Let $C$ be an irreducible non degenerate curve of degree $d$ in $\mathbb{P}^{r}$. Suppose that the regularity of $C$ is exactly $d-r+2$, then $C$ is either

1. a smooth rational curve having an extremal secant line,

2. or an elliptic normal curve

3. or a singular rational curve of degree $d$ in $\mathbb{P}^{d-1}$.

In the last two cases, $C$ has no extremal secant lines, i.e no 3-secant lines.

This has led us to check the regularity conjecture on varieties having an extremal secant line [1]. We have shown that smooth (not necessarily rational) scrolls satisfy the conjecture, using a similar argument to [6]. In particular, we have deduced that smooth varieties having an extremal secant line satisfy the conjecture. We show in this article that the same conclusion holds for singular varieties of codimension $e \geq 2$ having an extremal secant line.

To achieve this result, we'll need the following result of ours [1, which holds provided $X$ has an extremal secant line $l$ meeting $X$ at smooth points.

Theorem 2.4. Let $X$ be an irreducible, non degenerated variety of degree $d$ and codimension e in $\mathbb{P}^{r}$. Suppose that $X$ has an extremal secant line $l$, such that $l$ meets $X$ along smooth points of $X$. Then $X$ is the regular projection of a variety of minimal degree $\bar{X}$ of degree $d$ in $\mathbb{P}^{d+n-1}$.

Finally, we recall Bertini's famous irreducibility theorem in the form we shall use.

Theorem 2.5 (Bertini's irreducibility theorem). Let $X$ be a $n$-dimensional complex projective subvariety of $\mathbb{P}^{r}$ and $\mathcal{L}$ a non empty linear system on $X$. Suppose that $\mathcal{L}$ satisfies the following conditions

1. there exists a $n$-dimensional linear system $\mathcal{M}$ on $\mathbb{P}^{r}$ such that $\mathcal{L}=\left.\mathcal{M}\right|_{X}$ (if so one can find $\mathcal{M}$ satisfying moreover $\operatorname{dim}(\mathcal{M})=\operatorname{dim}(\mathcal{L})$ ), 
2. the image of $X$ by the rational map $\Phi_{\mathcal{L}}: X \rightarrow \mathbb{P}^{n}$ associated to $\mathcal{L}$ is a non degenerated subvariety $\mathbb{P}^{n}$ of dimension at least 2.

Then, the generic member of $\mathcal{L}$ is an irreducible and non multiple.

This theorem follows from Bertini's theorem on hyperplanes sections of $\Phi_{\mathcal{L}}(X)$ in $\mathbb{P}^{n}$ (for a modern proof see for instance 3] p 249) by duality. Remark that, if $X$ is non degenerated in $\mathbb{P}^{r}$ and $\Phi_{\Lambda}$ is a rational map on $X$ induced by a linear projection $\pi, \mathcal{L}$ satisfies the assumptions of theorem 2.5 provided that $\operatorname{dim}(\pi(X)) \geq 2$.

\section{The linear system of hyperplanes contain- ing an extremal secant line to $X$}

We need first to establish the following theorem which determines for which variety $X$ the image of $X$ by the linear system of hyperplanes passing through $l$ has dimension $<=1$, so that theorem 2.5 does not apply.

Theorem 3.1. Let $S$ be a non degenerate, irreducible surface of degree $d$ in $\mathbb{P}^{r}, r \geq 4$. Suppose that $l$ is an extremal secant line to $S$, such that the image of $S$ by the linear projection from $l$ is a curve $C$ in $\mathbb{P}^{r-2}$. Then $S$ is a cone $\langle p, \zeta\rangle$, where $p$ is a point and $\zeta$ is a smooth rational curve of degree $d$ in $\mathbb{P}^{r-1}$ having an extremal secant line $l^{\prime}$.

Proof. Let $q$ be a generic point on $l$; we denote by $\pi_{q}$ the projection from $q$. Let $q_{0} \in \mathbb{P}^{r-1}:=<q_{0},<C>>$ denote the image of $l$ by $\pi_{q}$. By dimension count, $\pi_{q}(S)=<q_{0}, C>$. Remark also that $S$ lies on the 3-dimensional cone $Y:=<l, C>$, as a sub-ruled surface, fibered in curves of degree $m:=$ $\operatorname{deg}\left(\pi_{q}\right)$. We have $\operatorname{deg}(C)=d / m$.

Lemma 3.2. The projection $\pi_{q}$ is generically one to one.

Proof. We wish to show that $m=1$. Let us desingularize $Y$ and compute the class of the strict transform of $S$ in this desingularization. Recall that the scroll $Y$ is the image of

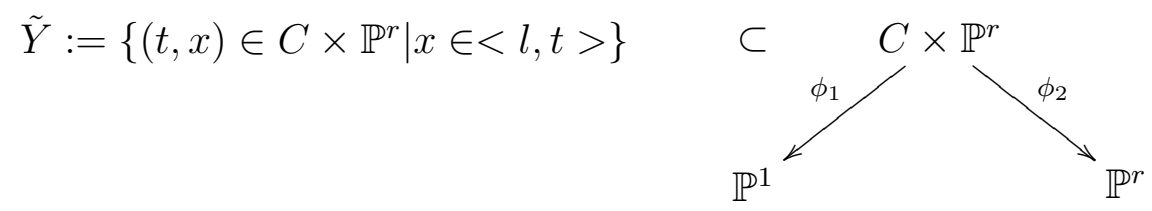


by the projection $\phi_{2}$ to $\mathbb{P}^{r}$. Let $\phi_{1}$ denote a generic linear projection of $C \subset \mathbb{P}^{r-2}$ onto $\mathbb{P}^{1}$. Let $\sigma$ be a fixed isomorphism $\mathbb{P}^{1} \stackrel{\sigma}{\rightarrow} l$. Consider the graph $\Delta:=\left\{(t, x) \in C \times l \mid \sigma \circ \phi_{1}(t)=x\right\}$; the projection map $\phi_{2}$ induces a degree $\operatorname{deg}(C)$-map from $\Delta$ onto $l$. If $C$ is smooth, $\tilde{Y} \stackrel{p}{\rightarrow} Y$, is a desingularization of $Y$ with exceptional locus $E:=C \times l$. Let $\bar{C} \stackrel{\nu}{\rightarrow} C$ be the normalization of $C$. The proper morphism $\bar{C} \times \mathbb{P}^{r} \stackrel{\left(\nu, i d_{\mathbb{P} r}\right)}{\longrightarrow} C \times \mathbb{P}^{r}$, induces a desingularization $\bar{Y} \stackrel{g}{\rightarrow} \tilde{Y}$ of $\tilde{Y}$, where $\bar{Y}$ denotes the strict transform of $\tilde{Y}$ by $\left(\nu, i d_{\mathbb{P}^{r}}\right)$. We have the following diagram

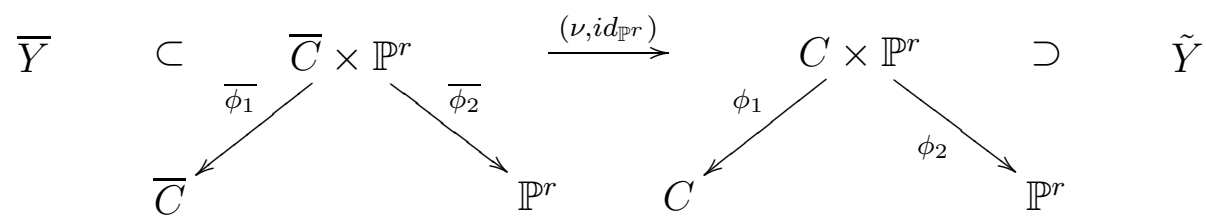

We have the following equations relating the projection maps:

$$
\overline{\phi_{2}}=\phi_{2} \circ\left(\nu, i d_{\mathbb{P}^{r}}\right)
$$

and

$$
\nu \circ \overline{\phi_{1}}=\phi_{1} \circ\left(\nu, i d_{\mathbb{P}^{r}}\right)
$$

It follows that $g$ satisfies $\left.\overline{\phi_{2}}\right|_{\bar{Y}}=\phi_{2} \circ g$. Since $\left.\overline{\phi_{2}}\right|_{\bar{Y}}$ and $\phi_{2}$ are projective, hence proper, the morphism $g$ is proper. The map $\bar{g}:=g \circ \phi_{2}$ induces a desingularization of $Y$. The Chow ring of $\bar{Y}$ is generated by $f$, the class of a fiber of the scroll $\bar{Y} \stackrel{\bar{\phi}_{1}}{\longrightarrow} \bar{C}$, and $h$ the class of restriction to $\bar{Y}$ of the pull back by $\overline{\phi_{2}}$ of an hyperplane in $\mathbb{P}^{r}$. From equation 2, we deduce that $f$ is also the class of the pull back by $g$ of a generic fiber of $\phi_{1}$. Moreover, equation 1, shows that $h$ is also the class of the pull back by $\bar{g}$ of hyperplane sections of $Y$ in $\mathbb{P}^{r}$. Those generators satisfy the obvious relations: $h^{4}=0, h^{3} f=0, f^{2}=0$. Let $\bar{S}$ be the strict transform of $S$ by $\bar{g}$. We have $[\bar{S}]=\alpha h+\beta f$, for some $\alpha, \beta \in \mathbb{Z}$. Since $g$ is proper and $\phi_{2}$ is projective, $\bar{g}$ is proper. The projection formula applied to $\bar{g}$, therefore shows

$$
\begin{gathered}
h^{3}-\operatorname{deg}(C) h^{2} f=0 \\
{[\bar{S}] \cdot h f=m} \\
{[\bar{S}] \cdot h^{2}=d}
\end{gathered}
$$

We thus find $\alpha=m$ and $\beta=0$. Let us compute the class of $\bar{\Delta}$, the strict transform of $\Delta$ by $g$. Applying the projection formula to $g$, we get $[\bar{\Delta}] \cdot f=1$, 
so that $[\bar{\Delta}]=h^{2}+b h f$ for some $b \in \mathbb{Z}$. Moreover, applying the projection formula to $\bar{g}$, we get $[\bar{\Delta}] \cdot h=(\operatorname{deg}(C))[l] \cdot h_{Y}$, where $h_{Y}$ denote the class of an hyperplane section of $Y$ in $C h o w\left(\mathbb{P}^{r}\right)$. Thus, $b=\operatorname{deg}(C) \mu$, where $\mu$ is the multiplicity at the point $q$, generic on $l$, of the intersection of $l$ with the cone $\langle q, C\rangle$. From the relation $\bar{\Delta} \cdot \tilde{S}=d-e+1$, we deduce that $m(1+\mu)=d-e+1$. Remark now that $\mu \leq \operatorname{deg}(C)-(e-1)+1$. Suppose that 1 is neither an extremal secant line nor a next to extremal secant line to $<q, C>$; we find $m(\operatorname{deg}(C)-(e-1)) \geq d-e+1$, so that $m(e-1) \leq e-1$. Since $e \geq 2$, it follows that $\delta=1$. Suppose that $l$ is a next to extremal secant line to $\langle q, C\rangle$; we get $m(e-2)=e-1$, this is clearly impossible. Finally, if $l$ is an extremal secant line to $\langle q, C\rangle$, we get $m(e-3)=e-1$. This has clearly no solutions for $e=3$. If $e \neq 3$ we find $\delta=1+\frac{2}{e-3}$, this is also impossible. Therefore, we have $m=1$, that is to say $\pi_{q}$ is birational.

From $m=1$, we deduce that $S$ is ruled in lines, $\gamma_{t}$, over $C$.

Lemma 3.3. The intersection $\langle q, C\rangle \cap X$ has dimension 1 . Let $\zeta$ be one of the 1-dimensional irreducible components of $\langle q, C\rangle \cap S$. The curve $\zeta$ projects from $q$ onto $C$.

Proof. We have $<q, C>\cap X=\cup_{t \in C}<q, t>\cap \gamma_{t}$. Therefore, $\operatorname{dim}(<q, C>$ $\cap S)=1$. Let $\zeta$ be a 1-dimensional irreducible component of $\langle q, C>\cap S$. Suppose, to the contrary, that $\pi_{q_{0}}(\zeta)$ is a point. By dimension count and irreducibility of $\pi_{q_{0}}(\zeta)$, we find $\zeta=<q_{0}, \pi_{q_{0}}(\zeta)>$. Since $\zeta \subset<q, C>, q_{0}$ lies on $<q, \pi_{q}(S)>$. Thus, $S \subset<q, q_{0}, C>=<q, \pi_{q}(S)>\subset \mathbb{P}^{r-1}$. This contradicts the non degeneracy of $S$.

The family of lines $\gamma_{t}$ can be therefore re-parameterized by $\zeta$. Let us denote by $\left\{p_{1}, \cdots, p_{s}\right\}$ the support of $l \cap S$. For $i \in\{1, \cdots s\}$, any point $p_{i}$ of $S \cap l$ must lie on a line $\gamma_{t}$, for some $t \in C$. It follows that $s=1$. Indeed, $S$ has dimension 2, so the following set is not empty:

$$
\mathcal{S}:=\left\{p \in\left\{p_{1}, \cdots, p_{s}\right\} \mid \exists U \subset X^{\prime \prime} \text { open, dense such that } \forall t \in U, p \in \gamma_{t}\right\}
$$

Suppose that $p_{1}$ belongs to $\mathcal{S}$. By irreducibility assumption, the variety $S$ must coincide with the cone $\left\langle p_{1}, \zeta\right\rangle$, since they intersect along a 2dimensional locus. The Castelnuovo-Mumford regularity of $S, \operatorname{reg}(S)$, satisfies $\operatorname{reg}(S) \geq d-e+1$, since $S$ has an extremal secant line. Besides, $\operatorname{reg}(S)=\operatorname{reg}(\zeta)$ for $X$ is a cone. Therefore, $\operatorname{reg}(S)=d-e+1=\operatorname{reg}(\zeta)$ and $\zeta$ is a curve of extremal regularity in $\mathbb{P}^{r-1}$. Applying theorem 2.3, we deduce 
that $\zeta$ is a smooth rational curve having an extremal secant line, an elliptic normal curve or a singular rational curve of degree $d$ in $\mathbb{P}^{d-1}(r=d)$.

Lemma 3.4. Suppose that $S$ in $\mathbb{P}^{r}$ is a cone $\langle p, C\rangle$, where $C$ is a curve in $\mathbb{P}^{r-1}$. Then, there exists an extremal secant line $l$ to $S$ through $p$ if and only if $C$ is a smooth rational curve having an extremal secant line.

Proof. Let $l$ be a secant line to $S$ through $p$. Let $x$ be the intersection of $l$ and $\langle C\rangle, x \notin C$. We can assume that $p$ has coordinates $(0: \cdots: 0: 1)$ and $x$ coordinates $(1: 0: \cdots: 0)$ in $\mathbb{P}^{d}$, so that $I_{l}:=\left(x_{1}, \cdots, x_{d-1}\right)$ is the saturated ideal defining the line $l$ in the polynomial ring $\mathbb{C}\left[x_{0}, \cdots, x_{d}\right]$. Let $I_{C}:=\left(f_{1}, \cdots, f_{t}\right)$ be the saturated ideal defining $C$ in $\mathbb{P}^{d-1}$; the scheme $l \cap S$ has defining ideal $\left(I_{C}+\left(x_{1}, \cdots, x_{d-1}\right)\right)=\left(f_{1}^{0}, \cdots f_{d-1}^{0}\right)+I_{l}$, where, for $i=1, \cdots, d-1$, the polynomial $f_{i}^{0}$ denotes the leading term of $f_{i}$ with respect to the lexicographic monomial ordering in $\mathbb{C}\left[x_{0}, \cdots, x_{d}\right]$. Therefore, the scheme $l \cap S$ has defining ideal $\left(f_{1}^{0}, \cdots, f_{d-1}^{0}\right)$ in $\mathbb{C}\left[x_{0}\right]$. The multiplicity of intersection of $l$ and $S$ at $p$ is therefore $\min \left\{d_{i} \mid f_{i}^{0} \neq 0\right\}$, where $d_{i}$ denote the degree of the polynomial $f_{i}$, for $i=1, \cdots, d-1$.

If $C$ is an elliptic normal curve or a singular curve of degree $d$ in $\mathbb{P}^{d-1}$, all the polynomials $f_{i}$ have degree 2 (see for instance 6]: $C$ satisfies property " $C_{2}$ " and is non degenerate), so that $l$ is only a bisecant line, while $d-$ $r+2=3$. If $C$ is a rational curve having an extremal secant line, we have $d_{i} \geq d-e+1$, so that $l$ is an extremal secant line to $S$.

Corollary 3.5. Let $X$ be a complex, irreducible non degenerate variety of degree $d$ and dimension $n \geq 2$ in $\mathbb{P}^{r}$. Assume that $X$ has an extremal secant line $l$. Moreover, suppose that the image of $X$ by the projection $\pi_{l}$ from $l$ is a variety $X^{\prime \prime}$ of dimension $n-1$. Then $X$ is a cone $\langle p, \zeta>$ over a $(n-1)$-dimensional variety $\zeta$ of $\mathbb{P}^{r-1}$.

Proof. Let $X$ in $\mathbb{P}^{r}$ be a variety satisfying the assumptions of theorem 3.1 We denote by $p_{1}, \cdots, p_{s}$ the support of $X \cap l$.

Lemma 3.6. Let $X$ be a variety satisfying the assumption of corollary 3.5. By induction on $n \geq 2$, the following properties hold:

1. $s=1$

2. For $q \in l \backslash\left\{p_{1}\right\}$ generic, the projection $\pi_{q}$ induces a birational map from $X$ onto its image. 
Proof. For $n=2$, the result follows from theorem 3.1. Suppose that the lemma holds for $(n-1)$-dimensional varieties such that $2 \leq n$. By assumption, the linear system of hyperplanes through $l$ cut on $X$ a linear system satisfying the assumptions of theorem 2.5, hence a generic hyperplane section $h_{1}$ of $X$ by a hyperplane $H$ through $l$ is an irreducible variety of dimension $n-1$, and degree $d$ to which $l$ is an extremal secant line.

By induction hypothesis, we deduce that $s=1$ and that ,for $q \in l \backslash\left\{p_{1}\right\}$, $\pi_{q}$ induces a birational map from $h_{1}$ onto its image. Let $t$ be a point of $X^{\prime \prime} \cap H$. This hyperplane $H$ contains the 2-plane $\langle t, l\rangle$, hence either it induces an hyperplane section of the fiber curve $\gamma_{t}$ of $X$ or $\gamma_{t}$ coincides with the line $H \cap\langle t, l\rangle$. Let $q \in l \backslash\left\{p_{1}\right\}$. By induction hypothesis, the projection $\pi_{q}$ induces a birational map from $h_{1}:=X \cap H$ onto its image, so that the ruled variety $h_{1}$ is ruled in lines over $\pi_{l}\left(h_{1}\right)$. The line $H \cap\langle l, t\rangle$ is one of the lines of the ruling of $h_{1}$. We deduce that $\gamma_{t}=H \cap\langle l, t\rangle$, so that $\pi_{q}$ induces a birational map from $X$ onto its image.

As in the 2-dimensional case, reparameterizing $X$ by any maximal dimensional component $\zeta$ of $<q, X^{\prime \prime} \cap X$ shows that $X$ is the cone $\left\langle p_{1}, \zeta\right\rangle$.

\section{Classification of varieties having an extremal secant line}

Going back to the inductive argument of lemma 1.1 in [1. We can correct it as follows.

Theorem 4.1. Let $X$ be a complex, irreducible, non-degenerate variety of degree $d$ and codimension e in $\mathbb{P}^{r}$, such that $X$ has an extremal secant line. Then, $X$ has an extremal secant line $l$ meeting $X$ along smooth points.

Proof. Let us proceed by induction on $n$. By theorem 2.3 the result is true for $n=1$. The result holds for $n=2$ by theorem 3.1

Suppose that the result holds for all integers $k$ such that $2 \leq k \leq n-1$. Let $X$ be a complex, irreducible, non-degenerate variety of degree $d$ and dimension $n$ in $\mathbb{P}^{r}$, such that $X$ has an extremal secant line $l$. Consider the linear system of hyperplane sections of $X$ by hyperplanes containing $l$.

Since $n \geq 3$, we can apply theorem 2.5 to this linear system. Its general member $h_{1}$ is thus irreducible and non multiple. The line $l$ is an extremal secant line to $h_{1}$. Indeed, let $H$ be the hyperplane cutting out $h_{1}$ on $X$. By 
construction, we have the following scheme inclusion: $H \cap X \supseteq l \cap X$. Thus, $l \cap X=l \cap h_{1}$.

Repeating the same argument for $h_{1}$, we find that the generic section $h_{n-2}$ of $X$ by $(e+2)$-planes containing $l$ is irreducible. The line $l$ is an extremal secant line for the surface $h_{n-2}$.

If the linear system of hyperplanes sections of $h_{n-2}$ passing through $l$ satisfies the assumptions of theorem 2.5, by theorem 2.3. the line $l$ meets a generic hyperplane section of $h_{n-2}$ by $l$ at smooth points. Therefore arguing as in [1, we deduce that $l$ meets $h_{n-2}$ at smooth points. Repeating the argument, the line $l$ meets $X$ along smooth points.

If $l$ doesn't meet $X$ along smooth points, the general surface section $h_{n-2}$ of $X$ by $(e+2)$-planes containing $l$ is a cone $\langle p, C\rangle$, where $C$ is a smooth rational curve of degree $d$ in $\mathbb{P}^{r-1}$ having an extremal secant line $l^{\prime}$. The line $l^{\prime}$ is an extremal secant line for $X$, since $l^{\prime} \cap h_{n-2} \subset l^{\prime} \cap X$ and length $\left(l^{\prime} \cap h_{n-2}\right)=d-e+1$. Repeating the previous argument with $l^{\prime}$ instead of $l$, we deduce that $l^{\prime}$ meets $X$ along smooth points.

We can now correct proposition 1 in [1]. The argument is as in [1, the crucial point is, of course, the irreducibility of the hyperplane section we use.

Corollary 4.2. Let $X$ be an irreducible non degenerate variety of degree $d$ and codimension $e \geq 2$ in $\mathbb{P}^{r}$. Suppose that $X$ has an extremal secant line $l$ meeting $X$ along smooth points of $X$. The image of $X$ by the projection $\pi_{l}$ from the line $l$ is a variety of minimal degree $X^{\prime \prime}$ in $\mathbb{P}^{r-2}$.

Proof. Let us proceed by induction on $n:=\operatorname{dim}(X)$. Notice that, to prove the first assertion, it is enough to show that $\operatorname{dim}\left(X^{\prime \prime}\right)=n$. Indeed, if it is so, the degree $d^{\prime \prime}$ of $X^{\prime \prime}$ satisfies : $d^{\prime \prime} \leq d-(d-r+n+1)=(r-$ $2)-n+1$. Suppose that $n=1$. Then, the property is trivially true, since $X$ is then a non degenerate non plane curve $(e \geq 2)$. Suppose that $n \geq$ 2. By assumption, the linear system of hyperplane sections through $l$ does satisfy Bertini's irreducibility theorem, since $X$ meets $l$ along smooth points. Therefore a generic member of the linear system of hyperplanes containing $l$, cuts $X$ along an irreducible non multiple variety. This linear system contains a pencil, since $r-2 \geq 1(e \geq 2$ and $n \geq 2)$. Let us thus consider two generic members $H_{1}$ and $H_{2}$ of such a pencil. By induction hypothesis, the varieties $X \cap H_{i}$, for $i=1,2$, are irreducible and satisfy the induction hypothesis. Their projection from $l$ is therefore $(n-1)$-dimensional. The space $\mathbb{P}^{r}$ is spanned by $<H_{1}, H_{2}>$ and $X$ is non degenerate, thus $X^{\prime \prime}$ is $n$-dimensional. 
Theorem 4.3. Let $S$ be a non-degenerate (possibly singular) surface of degree $d$ and codimension $e \geq 2$ in $\mathbb{P}^{r}$. Suppose that $S$ has a extremal secant line $l$, meeting $S$ along smooth points. Suppose that $S$ is the regular projection of a smooth rational normal scroll $\bar{S}$ of degree $d$ in $\mathbb{P}^{d+1}$. Then, $S$ is a smooth rational scroll.

Proof. Let $\Lambda$ denote the $(d-r)$-plane center of projection $\pi_{\Lambda}: \mathbb{P}^{d+1} \rightarrow \rightarrow \mathbb{P}^{r}$ mapping $\bar{S}$ to $S$. By assumption, we have $\Lambda \cap \bar{S}=\emptyset$. Thus, no curves on $\bar{S}$ can be contracted to a point by $\pi_{\Lambda}$. The $(d-r+2)$-plane $\bar{\Lambda}:=\langle\Lambda, l>$ is thus a $k$-secant plane to $\bar{S}$, for some integer $k \geq 1$.

Lemma 4.4. The $(d-r+2)$-plane $\bar{\Lambda}$ is a $(d-r+3)$-secant plane. Moreover, we have

$$
\mu_{x}(\Lambda \cap \bar{S}) \leq 2 \quad \forall x \in \bar{S}
$$

Proof. Let $\left\{\bar{p}_{1}, \cdots, \bar{p}_{m}\right\}$ denote the support of $\Lambda \cap \bar{S}$. Let $C$ be a hyperplane section of $S$ by a generic hyperplane of $\mathbb{P}^{r}$ containing $l$. Since $l$ meets $S$ at smooth points, $C$ is irreducible and $l$ is an extremal secant line to $C$. By theorem 2.3, $C$ is a smooth rational curve of degree $d$. Let $\bar{C}:=<$ $\Lambda, C>\cap \bar{S}$; it is a rational normal curve of degree $d$, projecting onto $C$ by $\pi_{\Lambda}$. By construction, the 0 -scheme $\bar{\Lambda} \cap \bar{S}$ is a subscheme of $\bar{\Lambda} \cap \bar{C}$. We have $\mu_{\bar{p}_{i}}(\Lambda \cap \bar{S}) \leq \mu_{\bar{p}_{i}}(\Lambda \cap \bar{C}) \leq 2$. Indeed, suppose to the contrary that $\mu_{\bar{p}_{i}}(\Lambda \cap \bar{C})>2$. Then, $\Lambda$ contains the projective tangent line $\mathbb{T}_{\overline{p_{i}}}(\bar{C})$ to $\bar{C}$ at $\overline{p_{i}}$, so that we would get

$$
\mu_{\bar{p}_{i}}(\Lambda \cap \bar{C})=\mu_{\bar{p}_{i}}\left(\mathbb{T}_{\bar{p}_{i}}(\bar{C}) \cap \bar{C}\right) \leq 2,
$$

for $\bar{C}$ has no 3 -secant line.

Claim 1. Let $p_{i}:=\pi_{\Lambda}\left(\overline{p_{i}}\right)$. We have

$$
\mu_{\overline{p_{i}}}(\bar{\Lambda} \cap \bar{C}) \geq \mu_{p_{i}}(C \cap l)
$$

Proof. The claim is trivially true if $\mu_{p_{i}}(C \cap l)=1$. Suppose that $\mu_{p_{i}}(C \cap l) \geq$ 2. The projectivized tangent line $\mathbb{T}_{\overline{p_{i}}}(\bar{C})$ is the limiting position of the lines $<\overline{p_{i}}, \bar{x}>$, for $x \in \bar{C} \backslash\{x\}$. Such a line cannot be contracted to a point by $\pi_{\Lambda}$, since $p_{i}$ is a smooth point of $C$. Therefore, the limiting position of $\pi_{\Lambda}(<$ $\left.\overline{p_{i}}, \bar{x}>\right)$ is $\mathbb{T}_{p_{i}}(C)$. By assumption, we have $l=\pi_{\Lambda}\left(\mathbb{T}_{\overline{p_{i}}}(\bar{C})\right)$, so that $\mu_{\overline{p_{i}}}(\bar{\Lambda} \cap$ $\bar{C})=2$. Let us show that we always have $\mu_{p_{i}}(C \cap l) \leq 2$. Up to a change of projective coordinates, we may assume that $p_{i}:=(0: 0: \cdots: 1)$ in $<$ $C>=\operatorname{Proj}\left(\mathbb{C}\left[x_{0}, \cdots, x_{r-1}\right]\right)$ and that the saturated ideal defining $l$ in $\langle C\rangle$ 
is $\left(x_{0}, \cdots, x_{r-3}\right)$. We write $\left\langle\bar{C}>=\operatorname{Proj}\left(\mathbb{C}\left[x_{r}, \cdots, x_{d}, x_{0}, \cdots, x_{r-1}\right]\right)\right.$ and denote by $I_{\bar{C}}$ the saturated ideal defining $\bar{C}$ in $\left\langle\bar{C}>\right.$. Let $\left\{\bar{f}_{1}, \cdots, \bar{f}_{u}\right\}$ be a Gröbner basis of $I_{\bar{C}}$ for lexicographic order on $\mathbb{C}\left[x_{r}, \cdots, x_{d}, x_{0}, \cdots, x_{r-1}\right]$. Since $\bar{C}$ is 2-regular, all the $f_{i}$ 's are degree 2-polynomials. Let us now proceed to the elimination; the subset $\left\{\bar{f}_{i_{1}}, \cdots, \bar{f}_{i_{v}}\right\}$ of $\left\{\bar{f}_{1}, \cdots, \bar{f}_{u}\right\}$ consisting of quadric polynomials in the variables $x_{0}, \cdots, x_{r-1}$ only is a Gröbner basis of $I_{C}$ for the lexicographic order on $\mathbb{C}\left[x_{0}, \cdots, x_{r-1}\right]$. For all $j \in\{1, \cdots, v\}$, let us write $\bar{f}_{i_{j}}=f_{i_{j}}^{0}+f_{i_{j}}^{1}$, where $f_{i_{j}}^{0}$ is a homogeneous polynomial in the variables $x_{r-2}$ and $x_{r-1}$ only. Then $\left(f_{i_{1}}^{0}, \cdots, f_{i_{v}}^{0}\right)$ is a defining ideal for the 0 -scheme $C \cap l$ in $l=\operatorname{Proj}\left(\mathbb{C}\left[x_{r-2}, x_{r-1}\right]\right)$; this scheme is supported at the point $(0: 1)$, hence the length of this 0 -scheme satisfies

$$
\mu_{p_{i}}(l \cap C)=\min \left\{\operatorname{deg}_{x_{r-2}}\left(f_{i_{j}}^{0}\right) \mid j \in\{1, \cdots, v\} \text { and } f_{i_{j}}^{0} \neq 0\right\},
$$

where $\operatorname{deg}_{x_{r-2}}\left(f_{i_{j}}^{0}\right)$ denotes the degree of the polynomial $f_{i_{j}}^{0}$, viewed as a polynomial in the variable $x_{r-2}$. Since $\operatorname{deg}_{x_{r-2}}\left(f_{i_{j}}^{0}\right) \leq \operatorname{deg}\left(f_{i_{j}}^{0}\right)=2, \forall j \in$ $\{1, \cdots, v\}$, we find $\mu_{p_{i}}(l \cap C) \leq 2$. This finishes the proof the claim.

Summing up the inequalities of the claim over the points of the support of the 0 -scheme $\bar{\Lambda} \cap \bar{C}$, we find $k \geq$ length $(\Lambda \cap \bar{C}) \geq d-r+3$. From theorem 2.1. we have $k \leq d-(d+1-2)+d-r+2=d-r+3$.

Let $s$ denote the number of points $y$ of $\bar{\Lambda}:=<\Lambda, l>$, such that $\mu_{y}(\bar{\Lambda} \cap \bar{S})=$ 2. We have $0 \leq s \leq \frac{d-r+3}{2}$. Let $\left\{\bar{p}_{1}, \cdots \bar{p}_{\delta-s}\right\}$ denote the support of the 0 scheme $\bar{\Lambda} \cap \bar{S}$. We may assume that $\mu_{\bar{p}_{i}}(\bar{\Lambda} \cap \bar{S})=2$ for $i \in\{1, \cdots, s\}$ and $\mu_{\bar{p}_{i}}(\bar{\Lambda} \cap \bar{S})=1$ otherwise.

Lemma 4.5. Let $S$ be a surface satisfying the assumptions of theorem 4.3. Suppose that $\delta-s \neq 3$ and $(\delta, s) \neq(4,2)$. Then, $S$ is smooth.

Proof. Let $x$ be a point of $S$. Let $\phi$ denote the regular map $\bar{S} \stackrel{\left.\pi_{\Lambda}\right|_{\bar{S}}}{\longrightarrow} S$. If $x \in S$ does not belong neither to the double locus nor to the ramification locus of $\phi, x$ is a smooth point of $X$. Suppose that $S$ is singular. Let $x$ be a singular point of $S$. Suppose first that the support of the fiber of $\phi$ at $x$ contains two distinct points $x_{1}$ and $x_{2}$. Let $\bar{C}$ be a section of $\bar{S}$ by some hyperplane, generic among hyperplanes containing $\bar{\Lambda}:=<\Lambda, l>$. We have $\bar{\Lambda}:=<\mathbb{T}_{\bar{p}_{1}}(\bar{C}), \cdots, \mathbb{T}_{\bar{p}_{s}}(\bar{C}), \overline{p_{\delta-2 s+1}}, \cdots, \overline{p_{\delta-s}}>$. Consider the following linear space

$$
L_{x}:=\left\{\begin{array}{l}
<\mathbb{T}_{\bar{p}_{1}}(\bar{C}), \cdots, \mathbb{T}_{\bar{p}_{s}}(\bar{C}), \overline{p_{\delta-2 s+1}}, \cdots, \overline{p_{\delta-s-2}}, \overline{x_{1}}, \overline{x_{2}}>\text { if } s \neq \delta / 2 \\
<\mathbb{T}_{\bar{p}_{1}}(\bar{C}), \cdots, \mathbb{T}_{\bar{p}_{s-1}}(\bar{C}), \overline{x_{1}}, \overline{x_{2}}>\text { if } s=\delta / 2
\end{array}\right.
$$


The linear space $\pi_{\Lambda}\left(L_{x}\right) \subset<l, x>$, is a line meeting $l$ at a single point, since $x \notin l$. Therefore, $\delta-s=3$ or $\delta=4$ and $s=2$. By assumption, this is not the case, hence the fiber of $\phi$ at a singular point of $S$ is supported at single point $\bar{x}$.

Consider a section $\overline{D_{x}}$ of $\bar{S}$ by some hyperplane $\bar{H}_{x}$ in $\mathbb{P}^{d+1}$, generic among the hyperplanes containing $\langle\Lambda, l\rangle$. Since $\bar{H}_{x}$ contains $\Lambda, H_{x}:=\pi_{\Lambda}\left(\bar{H}_{x}\right)$ is a hyperplane in $\mathbb{P}^{r}$. By Bertini's smoothness theorem, since $d+1-(d-$ $r+4)=e-1 \geq 1$, the degree $d$ curve $\overline{D_{x}}$ is smooth hence irreducible. The morphism $\phi_{\overline{D_{x}}}$ maps $\overline{D_{x}}$ to a curve $D_{x}$ on $S$, hyperplane section of $S$ be some hyperplane containing $\langle l, x\rangle$; hence, $\operatorname{deg}\left(D_{x}\right)=d$. The curve $D_{x}$ is the linear projection of an irreducible curve, hence is irreducible.

If $x$ doesn't belong to the ramification locus of $\left.\phi\right|_{\overline{D_{x}}}, x$ is a smooth point of $D_{x}$. Since $D_{x}$ is a Cartier divisor of $S, x$ is a smooth point of $S$. Therefore, $x$ is a ramification point of $\left.\phi\right|_{\overline{D_{x}}}$, i.e. $\mathbb{T}_{\bar{x}}\left(\overline{D_{x}}\right)$ meets $\Lambda$.

Consider the following linear space

$L_{x}:=\left\{\begin{array}{l}<\mathbb{T}_{\bar{p}_{1}}\left(\overline{D_{x}}\right), \cdots, \mathbb{T}_{\bar{p}_{s}}\left(\overline{D_{x}}\right), \overline{p_{\delta-2 s+1}}, \cdots, \overline{p_{\delta-s-2}}, \mathbb{T}_{\bar{x}}\left(\bar{D}_{x}\right)>, \text { if } \quad s \neq \frac{\delta}{2} \\ <\mathbb{T}_{\bar{p}_{1}}\left(\overline{D_{x}}\right), \cdots, \mathbb{T}_{\bar{p}_{s-1}}\left(\overline{D_{x}}\right), \mathbb{T}_{\bar{x}}\left(\bar{D}_{x}\right)>, \text { if } \quad s=\frac{\delta}{2}\end{array}\right.$

As in the previous case, the linear space $\pi_{\Lambda}\left(L_{x}\right) \subset<l, x>$, is a line meeting $l$ at a single point. Therefore, $\delta-s=3$ or $\delta=4$ and $s=2$. This contradicts our assumption. Thus, $S$ is smooth.

Lemma 4.6. Let $S$ be a surface satisfying the assumption of theorem 4.3. Suppose that $S$ is singular and $e \geq 3$.

1. Then, $S^{\prime \prime}$, its projection from $l$, is a surface $S(0, r-3)$, cone over a rational normal curve of degree $r-3$. All the singularities of $S$ lie in the 2 -plane projecting from $l$ onto the vertex of the cone $S^{\prime \prime}$. In particular, $S$ has isolated singularities.

2. Let $s$ denote the number of points $x \in \bar{\Lambda} \cap \bar{S}$ for which $\mu_{x}(\bar{\Lambda} \cap \bar{S})=2$. Then, we have $s \in 2 \mathbb{Z}_{\geq 1}$ and $\bar{S}=S(s / 2, d-s / 2)$.

Proof. Let us focus on the possible singularities of $S$. If $x \in S$ doesn't belong neither to the double locus nor to the ramification locus of $\pi_{\Lambda}, x$ is a smooth point of $S$, since $\bar{S}$ is smooth.

Suppose that two points $\alpha$ and $\beta$ get contracted to a double point $x$ of $S$. If the linear system $\mathcal{L}_{l, x}$ of hyperplane section of $S$ by hyperplanes of $\mathbb{P}^{r}$ 
through $\langle l, x\rangle$ satisfy the assumption of theorem 2.5. a general member of $\mathcal{L}_{l, x}$ is a singular irreducible curve having an extremal secant line. This contradicts theorem 2.3. Therefore, the projection of $X$ from the 2-plane $\langle l, x\rangle$ is a curve $C$ in $\mathbb{P}^{r-3}$, i.e. the surface $S^{\prime \prime}:=\pi_{l}(S)$ is the cone $<\pi_{l}(x), C>$ over a rational normal curve $C$ of degree $r-3$.

Suppose that $\langle\Lambda, y\rangle$ contains a tangent line $T$ to $\bar{S}$ at $y$, i.e $x:=\pi_{\Lambda}(y)$ belongs to the ramification locus of $\pi_{\Lambda}$. We write $\bar{\Lambda}:=<\Lambda, l>$. Then, unless the linear system of hyperplane sections through $\langle\bar{\Lambda}, y\rangle$ doesn't satisfy assumptions of theorem 2.5, i.e. $X^{\prime \prime}=\langle x, C>$ for a rational normal curve $C$ of degree $r-3$, a generic hyperplane section through $\langle\bar{\Lambda}, y\rangle$ is a rational normal curve $D$ tangent to $x$ along the line $T$. Therefore, $x$ is a smooth point of the linear section of $X, \pi_{\Lambda}(D)$, for this curve has an extremal secant line $l$. Thus, $x$ is a smooth point of $S$. In summary, if $x$ is a singular point of $S$, then $S^{\prime \prime}$ is a cone $\langle q, C\rangle$ over a rational normal curve of degree $r-3$, and $\pi_{l}(x)=q$.

Let $\left\{\bar{p}_{1}, \cdots, \bar{p}_{m}\right\}$ denote the support of $\bar{\Lambda} \cap X$, where $m \leq d-e+1$. If $s$ denotes the number of points $x \in \bar{\Lambda} \cap \bar{S}$ for which $\mu_{x}(\bar{\Lambda} \cap \bar{S})=2$, we have $m=\delta-s$, where $\delta:=d-r+3$. Since $\bar{S}$ is smooth, for $i \neq j \in\{1, \cdots, m\}$, we have $\bar{p}_{j} \notin \bar{f}_{i}$, where $\bar{f}_{i}$ denotes the fiber of $\bar{S}$ passing through $\bar{p}_{i}$

The projection $\pi_{\bar{\Lambda}}$ from $\bar{S}$ to $S^{\prime \prime}$, induces an elementary transformation at $\bar{p}_{1}, \cdots, \bar{p}_{m}$ from $\bar{X}$ to the surface $\mathbb{F}_{r-3}$, blowing up of the cone $S^{\prime \prime}$ at its vertex $q$.

Indeed, the indeterminacy locus of the rational map induced by $\pi_{\bar{\Lambda}}$ on $\bar{S}$ is resolved by blowing up $\bar{p}_{1}, \cdots, \bar{p}_{m}$. Let $\tilde{S}$ denote the blowing up of $X$ at $\bar{p}_{1}, \cdots, \bar{p}_{m}$ and, for $i=1, \cdots, m$, let $E_{i}$ denote the exceptional curve over the point $p_{i}$ and $\tilde{f}_{i}$ the strict transform of the fiber of $\bar{f}_{i}$.

The resolving regular map $\phi$ from $\tilde{S}$ to $S^{\prime \prime}$ is associated to a sub-system of the complete linear system $\left\|H-\sum_{i=1}^{m} E_{i}\right\|$, so that it contracts $\tilde{f}_{i}$ to a point of $X^{\prime \prime}$ and sends $E_{i}$ to a line of $X^{\prime \prime}$.

Since $\operatorname{deg}(C) \geq 2$, the only lines of $\langle q, C\rangle$ are lines joining $q$ to a point of $C$, i.e. blow-down of fibers of the scroll $\mathbb{F}_{r-3}$. The birational map from $\bar{S}$ to $\mathbb{F}_{r-3}$, induced by $\pi_{\bar{\Lambda}}$ factors though an elementary transformation $\sigma$ of $\bar{S}$ at $\overline{p_{1}}, \cdots, \overline{p_{m}}$ and a composition of blow-downs of $(-1)$-curves to $\mathbb{F}_{r-3}$. Let $\Sigma$ be the smooth rational ruled surface image of $\bar{S}$ by $\sigma$. The surface $\Sigma$ is isomorphic to a surface of type $\mathbb{F}_{n}$ for some integer $n \geq 0$. If $n \neq 1$, by minimality of $\mathbb{F}_{n}$ we get $n=r-3$. If $n=1$, then $\Sigma$ has a unique $(-1)$-curve, which gets blown-down to a point of $\mathbb{F}_{r-3}$; thus, $\mathbb{F}_{0} \simeq \mathbb{P}^{2}$ is an 
elementary transformation of $\mathbb{F}_{r-3}$ at a point $y$ of $\mathbb{F}_{r-3}$, so that $r-4=0$ or $r-2=0$. From the assumption $e \geq 3$, neither of the two cases can happen. In conclusion, $\Sigma$ is isomorphic to $\mathbb{F}_{r-3}$ and $\phi$ is an elementary transformation of $\bar{X}$ at $\overline{p_{1}}, \cdots, \overline{p_{m}}$.

Claim 2. Let $\psi$ denote the birational map from $\bar{S}$ to $X^{\prime \prime}=\langle q, C\rangle$, induced by $\pi_{\bar{\Lambda}} ; \psi^{-1}$ is defined away from the vertex of the cone $q$.

Proof. If $x \in S$ is a smooth point, we denote by $f_{x}$ the image by $\pi_{\Lambda}$ of the fiber of $\bar{S}$ at the preimage of $x$ by $\pi_{\Lambda}$. Since $\Lambda \cap \bar{S}=\emptyset$, no fibers of $\bar{S}$ can be contracted to a point by $\pi_{\Lambda} ; f_{x}$ is thus a line. We call $f_{x}$ the fiber of $S$ at $x$.

Since $\psi$ induces an elementary transformation at $\overline{p_{1}}, \cdots, \overline{p_{m}}$, it is enough to show that for all $i=1, \cdots, m$ the fibers of $S$ at $p_{i}$ are contracted by $\psi$ to $q$.

Suppose, to the contrary, that there exist $i \in\{1, \cdots, m\}$ such that the fiber of $S$ at $p_{i}$ is contracted to $z \neq q$ on $\langle q, C\rangle$. Pick a generic point $y$ on the line $\left\langle q, z>\right.$; since $y \neq q, y$ is the image by $\pi_{l}$ of a smooth point $x$ of $S$. Since $y$ is generic on $\langle q, z\rangle$, for all $j \in\{1, \cdots, m\}$ we have $f_{x} \neq f_{p_{j}}$, so that $f_{x}$ meets the 2-plane $<l, f_{p_{i}}>$ transversally at some point $x^{\prime}$. Since $\pi_{l}\left(x^{\prime}\right)=z \neq q, x^{\prime}$ is a smooth point of $S$, hence cannot lie on the fiber $f_{p_{i}}$. Remark that the generic member of the linear system of hyperplane sections through $l$ and $x^{\prime}$ is not irreducible. Thus, $\pi_{z}(<q, C>)$ is a curve $C^{\prime}$ of $\mathbb{P}^{r-3}$. The cone $\left\langle q, C>\right.$ thus coincides with the cone $\left\langle z, C^{\prime}\right\rangle$. Since $\operatorname{deg}(C)=r-3 \geq 2, q=z$ and we get a contradiction.

Let $\phi$ be the birational inverse of $\psi$; it is an elementary transformation at $m$ distinct points $q_{1}, \cdots, q_{m}$ of $\mathbb{F}_{r-3}$.

Since the inverse of $\psi$ is defined away from the vertex of cone $\langle q, C\rangle$, the $m$ points $q_{1}, \cdots, q_{m}$ must lie on the unique $(-(r-3))$ - curve on $\mathbb{F}_{r-3}$, $E_{q}$, the exceptional locus of the blow-up of the cone $\langle q, C\rangle$ at its vertex $q$. By the blowing-down of the fibers $g_{i}$ of the points $q_{i}$ for $i=1, \cdots, m$, $E_{q}$ is blown down to a $(-(r-3+m))$ - irreducible curve on $\bar{S}$. We write $\bar{S}:=S(\alpha, \beta)$, with $1 \leq \alpha \leq \beta$. Since $\bar{S} \simeq \mathbb{F}_{\beta-\alpha}$, we find $\beta-\alpha=r-3+m$. From $\alpha+\beta=d$, we find $s \in 2 \mathbb{Z}_{\geq 1}$ and $\alpha=s / 2 \beta=d-s / 2$.

The singular locus of $S$ lies in the 1-dimensional intersection of $S$ with the 2-plane $<l, q>$; since $l \cap S$ is supported at smooth points of $S, \operatorname{Sing}(S)$ is 0 -dimensional. 
If $\delta-s=3$, we find $s=0$ or $s=1$. Thus, combining lemma 4.5 and 4.6, we deduce that, if $S$ is singular, either $e \geq 3, \delta=4$ and $s=2$, or $e=2$ and $\delta-s \in\{2,3\}$.

Lemma 4.7. Suppose that $S$ satisfies the assumptions of theorem 4.3. If $e=2, S$ is smooth.

Proof. By lemma 4.5. we can assume that $\delta-s=3$ or $\delta=4$ and $s=2$. That is to say $(d, \delta, s) \in\{(5,4,2),(5,4,1),(4,3,0)\}$. Suppose first that $(d, \delta, s)=$ $(4,3,0)$. Then, we have $r=4=d$. By corollary 4.2, $\pi_{l}(S)=\mathbb{P}^{2}$. Hence for any point $x$ of $S, \pi_{l, x}(S)=\mathbb{P}^{1}$. In particular, the generic member $D_{x}$ of the linear system cut out on $S$ by hyperplanes through $\langle x, l\rangle$ is irreducible, since generic hyperplane sections of $\mathbb{P}^{1}$ are irreducible. Suppose first that $(d, \delta, s)=(4,3,0)$. Then, we have $r=4=d$, so that $D_{x}$ is a rational quartic in $\mathbb{P}^{3}$ having a 3 -secant line $l$. By theorem 2.3 $x$ is a smooth point of $D_{x}$. The point $x$ is thus a smooth point of $S$, for $D_{x}$ is a Cartier divisor on $S$. The surface $S$ is thus smooth.

If $(d, \delta, s) \in\{(5,4,2),(5,4,1)\}$, we find $d=5=r-1$, so that $D_{x}$ is either a possibly singular curve of degree 5 in $\mathbb{P}^{3}$. If $\operatorname{dim}\left\langle D_{x}\right\rangle=2$, $D_{x}$ is a plane quintic curve, so that any line meets $D_{x}$ with multiplicity $\operatorname{deg}\left(D_{x}\right)=5>\delta=4$. This gives a contradiction, since, by construction, $D_{x} \cap l=S \cap l$ as 0 -schemes. Therefore, $\operatorname{dim}\left\langle D_{x}\right\rangle=3$, and $D_{x}$ has an extremal secant line $l$. The curve $D_{x}$ is thus smooth, showing that $x$ is a smooth point of $S$.

The only remaining case is $e \geq 3$ and $(\delta, s)=(4,2)$, i.e. $(d, \delta, s)=$ $(e+3,4,2)$ and $d \geq 6$. Suppose that $S$ is singular. In particular, $\pi_{l}(S)=$ $S(0, r-3)$. We have $\bar{S}=S(1, d-1)$. So that there is a line $l^{\prime}$ on $\bar{S}$ meeting every fiber $f_{x}$ of $\bar{S}$ at a single point $y(x)$; re-parameterizing $\bar{S}$ by $l^{\prime}$, we can assume $x=y(x)$. Since $\Lambda$ doesn't contract any curve on $\bar{S}, \pi_{\Lambda}\left(l^{\prime}\right)$ is a line on $S$, meeting $\pi_{\Lambda}\left(f_{x}\right)$ for all $x \in l^{\prime}$. Suppose that $\operatorname{dim}\left(\pi_{l}\left(\pi_{\Lambda}\left(l^{\prime}\right)\right)\right)=1$, then $\left.\pi_{l}\left(\pi_{\Lambda}\left(l^{\prime}\right)\right)\right)$ is a line on $S(0, r-3)$ meeting all lines $\pi_{\bar{\Lambda}}\left(f_{x}\right)$ for all $x \in l^{\prime} \backslash l$. Since $r-3 \geq 2$,i.e. $S(0, r-3) \neq \mathbb{P}^{2}$; this is impossible. Therefore, $l^{\prime}$ gets contracted by $\pi_{\bar{\Lambda}}$ to a point on the cone $S(0, r-3)$. Let us consider the elementary transformation at $\left\{\bar{p}_{1}, \bar{p}_{2}\right\}$ from $S(1, d-1)$ to $\mathbb{F}_{r-3}$ induced by $\left.\pi_{\bar{\Lambda}}\right|_{\bar{S}}$. Let $\tilde{S}$ denote the blow up of $\bar{S}$ at $\left\{\bar{p}_{1}, \bar{p}_{2}\right\}$ and $E_{1}, E_{2}$ the exceptional divisors on $\tilde{S}$ over $\bar{p}_{1}$ and $\bar{p}_{2}$ respectively. We have $\operatorname{Pic}(\tilde{S})=\mathbb{Z} H^{*} \oplus \mathbb{Z} f^{*} \oplus \mathbb{Z} E_{1} \oplus \mathbb{Z} E_{2}$, where $H^{*}$ is the pull-back of the class $H$ of hyperplane sections in $S(1, d-1)$ and $f^{*}$ is the pull-back of the class of a fiber in $S(1, d-1)$. Recall that 
$\left[l^{\prime}\right]=H-(d-1) f$ in $\operatorname{Pic}(\bar{S})$, so that the class of the strict transform $\tilde{l}^{\prime}$ of $l^{\prime}$ in $\tilde{S}$ is of the form $H^{*}-(d-1) f^{*}-\epsilon_{1} E_{1}-\epsilon_{2} E_{2}$, with $\epsilon_{i} \in\{0,1\}$ for $i=1,2$. Moreover, since $\pi_{\Lambda}\left(l^{\prime}\right) \neq l$, we get $\epsilon_{1}+\epsilon_{2} \leq 1$. If $\psi$ contracts $l^{\prime}$ to a point, we have $\left(\tilde{l}^{\prime}\right)^{2}=-1$. Since $\left(\tilde{l}^{\prime}\right)^{2}=-d+2+\epsilon_{1}+\epsilon_{2}$, this implies that $d \in\{3,4\}$. Since $d=e+3 \geq 6, \psi$ cannot contract $l^{\prime}$. Therefore, since $\pi_{\bar{\Lambda}}\left(l^{\prime}\right)$ is a point on $S(0, r-3), \psi\left(l^{\prime}\right)=\gamma$, where $\gamma$ is the unique irreducible curve on $\mathbb{F}_{r-3}$, such that $\gamma^{2}=-(r-3)$. Therefore $\tilde{\gamma}=\tilde{l}^{\prime}$. We find, $-(r-3)+2=-d+1+\epsilon_{1}+\epsilon_{2}$, so that

$$
\epsilon_{1}+\epsilon_{2}-1=d-(r-3)=\delta=4 \text {. }
$$

This contradicts trivially $\epsilon_{1}+\epsilon_{2} \leq 1$. Therefore, $S$ is smooth.

Corollary 4.8. Let $X$ be a non-degenerate complex complex projective variety of degree $d$ and codimension $e \geq 2$ in $\mathbb{P}^{r}$. Suppose that $X$ has an extremal secant line $l$. Then $X$ is either

- a cone $\langle L, V\rangle$, where $L$ is a linear space of dimension $k \geq-1$ and $V$ is the Veronese surface in $\mathbb{P}^{5}$,

- a cone $\left\langle L, V^{\prime}\right\rangle$, where $L$ is a linear space of dimension $k \geq-1$ and $V^{\prime}$ is the isomorphic projection of the Veronese surface to $\mathbb{P}^{4}$,

- a cone $<L, X_{0}>$, where $L$ is a linear space of dimension $k \geq-1$ and $X_{0}$ is either a smooth rational scroll with an extremal secant line or a smooth rational curve with an extremal secant line,

Proof. If $X$ is smooth this is a consequence of our previous results [1].

Suppose that $X$ is singular.

By theorem 4.1, we can assume that there exists an extremal secant line $l$ to $X$ meeting $X$ along smooth points. Applying theorem 2.4, we deduce that $X$ is the regular projection of a variety of minimal degree $\bar{X}$ in $\mathbb{P}^{d+n-1}$.

Let us first assume that $\bar{X}$ is smooth. By theorem $2.2(e \geq 2), \bar{X}$ is either the Veronese surface $V$ in $\mathbb{P}^{5}$ or a smooth rational normal scroll.

Lemma 4.9. Let $X$ satisfying the assumption of theorem 4.8. Suppose that $\bar{X}$ is smooth. Then $X$ is either the Veronese variety $V$ in $\mathbb{P}^{5}, V^{\prime}$ its isomorphic projection to $\mathbb{P}^{4}$, or a smooth rational scroll $X_{0}$ having an extremal secant line.

Proof. Let us first assume that $\bar{X}=V$. Suppose $\bar{X} \neq X$. The regular projection to $X$ in $\mathbb{P}^{r}$, is either an isomorphism and $X=V^{\prime}$ or $X$ is the Steiner surface in $\mathbb{P}^{3}$. The last case can be ruled out, since $e \geq 2$. 
Suppose now that $\bar{X}$ is a smooth rational normal scroll and assume that $X \neq \bar{X}$. If $X$ is singular, the singularities of $X$ were created by the projection.

The lemma is true if $n=2$ (theorem 4.3), so we can assume $n \geq 3$. Let us prove by induction on $n \geq 2$ that $X$ is smooth. Suppose that the lemma is true for $n-1 \geq 2$. Let $x$ be a point of $X \backslash l$. We wish to show that $x$ is a smooth point of $X$. Let $h_{1}$ be an hyperplane section of $X$ by a generic hyperplane $H$ through $\langle l, x>$.

Suppose that $h_{1}$ is not irreducible; then, $\pi_{\pi_{l}(x)}\left(X^{\prime \prime}\right)$ is a curve $C$, so that $X^{\prime \prime}$ is the 2-dimensional cone $\left\langle\pi_{l}(x), C\right\rangle$. This contradicts corollary 4.2. the hyperplane section $h_{1}$ is thus irreducible.

The line $l$ is an extremal secant line to $h_{1}$. Moreover, the variety $h_{1}$ is the projection of the smooth rational normal scroll $\overline{h_{1}}$, hyperplane section of $\bar{X}$ by the hyperplane $\langle\Lambda, H\rangle$. By induction hypothesis, $h_{1}$ is smooth, so that $x$ is a smooth point of $h_{1}$. Since $h_{1}$ is a Cartier divisor on $X$, the point $x$ is a smooth point of $X$.

Only remains the case $\bar{X}$ singular. By theorem $2.2, \bar{X}$ is a cone $<L, \tilde{X}\rangle$, where $L$ is a linear space of arbitrary dimension and $\tilde{X}$ is a smooth rational normal scroll. Consider again the center of projection $\Lambda$, of the regular projection mapping $\bar{X}$ to $X$. Notice that, since $\Lambda \cap X=\emptyset$, no lines $\langle p, q\rangle$ joining a point of $p$ of $\tilde{X}$ to a point $q$ of the vertex of the cone $L$ can be contracted to a point by the projection to $X$.

Thus, the cone $\langle L, \tilde{X}\rangle$, projects by $\pi_{\Lambda}$ onto a cone $\left\langle L^{\prime}, X_{0}\right\rangle$, where the rational scroll $X_{0}$ is the image of $\tilde{X}$ by $\pi_{\Lambda}$. From the inequality

$\operatorname{dim}\left(L^{\prime}\right)+\operatorname{dim}\left(X_{0}\right)+1=\operatorname{dim}(X) \leq \operatorname{dim}(L)+\operatorname{dim}(\tilde{X})+1=\operatorname{dim}(\bar{X})=\operatorname{dim}(X)$

we deduce that $\operatorname{dim}\left(L^{\prime}\right)=\operatorname{dim}(L)$. By proposition 4.9, if $X_{0}$ has an extremal secant line, $X_{0}$ is smooth since it is the projection of a smooth rational normal scroll.

So we may assume that $l$ is not an extremal secant line to $X_{0}$, i.e. $l$ does not lie in the linear space $\left\langle X_{0}\right\rangle$. Since $l$ meets $X$ along smooth points of $X, l \cap L=\emptyset$. Moreover, we have $l \cap X_{0}=\emptyset$.

Indeed, suppose, to the contrary, that there exists a point $p \in l \cap X_{0}$. Since $l \not \subset<X_{0}>$, the line $l$ meets $X$ at another point $q$ of $X \backslash\left(L \cup X_{0}\right)$. The line $l=<q, p>$ therefore lies in the $(k+1)$-plane $\langle p, L>$ and meets $L$ at some point $x \in L$. Thus, $l$ coincides with the ruling line $\langle p, x>\subset X$. This gives a contradiction. 
Therefore, our extremal secant line $l$ to $X$ is the projection of a curve in $\langle\Lambda, l\rangle$ meeting $\bar{X}$ away from $L$ and $\tilde{X}$. Let us rule out this case. First, notice that from corollary 4.2 the variety $X$ projects from $l$ onto a variety $X^{\prime \prime}$ of minimal degree $d$ in $\mathbb{P}^{r-2}$.

Let us first assume that $l \cap\left\langle X_{0}\right\rangle \neq \emptyset$; we denote by $x$ the intersection point $l \cap\left\langle X_{0}\right\rangle$. Notice that $\pi_{l}\left(X_{0}\right)$ is a variety of minimal degree. Indeed, the variety $\pi_{l}(X)$ is the join variety $\left\langle\pi_{l}(L), \pi_{l}\left(X_{0}\right)>\right.$, which turns out to be a variety of minimal degree of dimension $n$ and degree $d$ in $\mathbb{P}^{r}$. Since $l \cap L=\emptyset$, we can realize $\pi_{l}$ as a projection onto the $(r-2)$-plane $\left\langle L, \pi_{l}\left(<X_{0}\right\rangle\right)>$, so that $\pi_{l}(L)=L$ and $\pi_{l}(X)$ is the cone $<L, \pi_{l}\left(X_{0}\right)>$. Therefore, $\pi_{l}\left(X_{0}\right)$ is a variety of degree $d$, dimension $\operatorname{dim}\left(X_{0}\right)=n-\operatorname{dim}(L)$ and the same codimension in $\left\langle\pi_{l}\left(X_{0}\right)>\right.$ as $\pi_{l}(X)$ in $\left\langle\pi_{l}(X)\right\rangle$, hence $\pi_{l}\left(X_{0}\right)$ is a variety

of minimal degree. Let $q$ be a generic point on $l$, we can realize $\pi_{q}$ as a projection from $\mathbb{P}^{r}$ onto the $(r-1)$-plane $<<X_{0}>, \pi_{q}(L)>$. Therefore, its restriction to $X_{0}$ is the identity map. Since $\pi_{l}=\pi_{x} \circ \pi_{q}$, the projection $\pi_{x}$ induces a birational map from $X_{0}$ onto its image $\pi_{l}\left(X_{0}\right)$. Since $\pi_{l}\left(X_{0}\right)$ is a variety of minimal degree, it is linearly normal, so that $X_{0}=\pi_{l}\left(X_{0}\right)$, i.e. $x \notin<X_{0}>$.

Since $\left.l \cap<X_{0}\right\rangle=\emptyset$, the the projection $\pi_{l}$ induces the identity map between $X_{0}$ and its image $X_{0}^{\prime \prime}$, which is a variety of minimal degree $d$. Therefore, $X$ is a variety of variety of minimal degree. This contradicts $\bar{X} \neq X$.

Since a cone has the same regularity as its base and smooth scrolls satisfy the regularity conjecture [1], if $e \geq 2$, varieties having an extremal secant line satisfy the regularity conjecture.

Acknowledgements I am grateful to Atsushi Noma for pointing out this mistake in my previous paper.

\section{References}

[1] Bertin M.-A. On the regularity of varieties having an extremal secant line. Jour. für die reine und angew. Math 2002, 545, 167-181.

[2] Castelnuovo G. Memorie Scelte; N.Zanichelli: Bologna, 1937. 
[3] Danilov V.I. Algebraic varieties and schemes. In Algebraic Geometry I; Shafarevich I. Eds. ; Encyclopaedia of Mathematical Sciences; Springer Verlag: 1984; vol 23, 167-307.

[4] Eisenbud D.; Goto S. Linear free resolutions and minimal multiplicity. Jour. of Algebra 1984, 88, 89-133.

[5] Eisenbud D. ; Harris J. Varieties of minimal degree (a centenial account). In Bowdoin conference in Algebraic Geometry, Brunswick, Maine, 1985; Proc. Symp. Pure Math., 1987, XXXXVI, part I , 1-13.

[6] Gruson L; Lazarsfeld R.; Peskine C. On a theorem of Castelnuovo and the equations defining space curves. Math. Inv., 1983, 72, 491-506.

[7] Kwak S. Smooth projective varieties with extremal or next to extremal curvilinear spaces, to appear in Transactions of the AMS

[8] Lazarsfeld R. A Castelnuovo bound for smooth surfaces. Duke Math. J., 1987, 55, 423-429.

[9] Noma A. results announced at the 150th birthday of Veronese in Siena (June 2004).

[10] Pinkham H. Castelnuovo bound for smooth surfaces. Inv. Math, 1986, 83. 